CHAPITRE 2

\title{
LA GÉNÉTIQUE DES CARACTÈRES QUANTITATIFS : MÉTHODES D'ANALYSE ET POSSIBILITÉS D'UTILISATION
}

\author{
Francine KRIEG
}

Laboratoire de génétique des poissons, INRA - 78350 JOUY-EN-JOSAS

\section{INTRODUCTION}

On appelle caractère quantitatif un caractère mesurable dont la variation est continue. Ce caractère peut donc prendre une infinité de valeurs possibles (par exemple le poids, la longueur...). On étend cette définition à des caractères s'exprimant par un nombre entier, mais présentant plusieurs valeurs possibles comme la fécondité ou le nombre d'écailles. On considère alors ces mesures comme corrrespondant à un caractère dont la variation est continue mais s'exprimant par un nombre entier.

L'expression de ces caractères résulte généralement d'interactions complexes entre de nombreux facteurs de l'environnement et le programme génétique de l'animal, lui-même constitué d'un grand nombre de gènes s'exprimant successivement ou simultanément. L'observation d'un caractère donné chez un individu ou une population (le phénotype) ne permet pas d'en déduire simplement la "valeur génétique" (le génotype) de cet individu. Cependant, à la suite des travaux de FISHER (1918), des modèles d'analyse se sont développés pour répondre à ce problème. Ils permettent, sans connaître précisément les gènes en cause et leurs effets individuels, de cerner les sources majeures de variation d'un caractère quantitatif. Les ouvrages de base de FALCONER (1960) pour la théorie générale et de KIRPICHNIKOV (1981) pour le cas de poissons font de ce problème une présentation détaillée. Nous exposerons ici les grandes lignes de ces méthodes et leurs applications à la gestion génétique des populations.

\section{LES MÉTHOdES D'ANALYSE DE LA VARIATION QUANTITATIVE}

\section{La description de la variabilité phénotypique}

A partir de la mesure d'un nombre élevé d'individus, on peut décrire la variation phénotypique au sein d'une population par une distribution. Pour pouvoir conduire une analyse statistique satisfaisante, cette distribution doit être relativement voisine d'une distribution théorique normale. C'est par exemple le cas de la longueur dans une population de truites (figure 1). Dans le cas contraire, une transformation mathématique normalisante doit être appliquée. Certaines distributions restent cependant extrêmement différentes de la distribution normale. Par exemple la longueur de jeunes saumons atlantiques d'un an (figure 2), où on observe deux groupes très différents correspondant aux individus smoltifiants et non smoltifiants. Dans ce cas, il convient d'analyser séparément ces deux populations; la longueur moyenne n'ayant aucun sens biologique.

Lorsque le caractère est "qualitatif", comme la "robe" d'une truite, le "goût" de sa chair ou sa "combativité", il ne peut être traité par le généticien que si celui-ci dispose d'une échelle de mesure objective. Or, la définition de cette mesure est parfois difficile: plusieurs mesures peuvent être proposées et le choix qui sera fait peut très bien conduire à des résultats relativement différents sur le plan de l'analyse génétique. Ainsi, un critère "simple" comme la couleur de la chair nécessite trois paramètres physiques pour être défini (luminosité, longueur d'onde dominante et pureté d'excitation); ces paramètres pouvant varier de manière relativement indépendante d'un groupe à l'autre (CHOUBERT et BLANC, 1985).

\section{Effets génétiques et environnementaux}

Le phénotype d'un individu résulte de la combinaison de facteurs génétiques et environnementaux. Pour une analyse génétique du caractère, il est nécessaire, au moins dans un premier temps, de supposer l'additivité de ces facteurs; autrement dit, un milieu donné agit de la même façon sur des génotypes variés. Cependant l'utilisation de ces résultats dans des milieux différents implique nécessairement de valider ces mêmes résultats dans chaque milieu. Citons pour exemple l'étude de MOAV et al. (1975) qui comparent plusieurs populations de carpe dans des milieux différents (figure 3). Suivant le milieu, la population choisie ne sera pas la même.

Cependant, en admettant l'additivité comme postulat de départ, on écrit :

$$
P=G+E
$$

d'où en termes de variance : $V_{P}=V_{G}+V_{E}$

Pour séparer strictement ces deux composantes, il faudrait:

- supprimer $V_{G}$ en utilisant des individus génétiquement identiques: cela peut être réalisé chez 


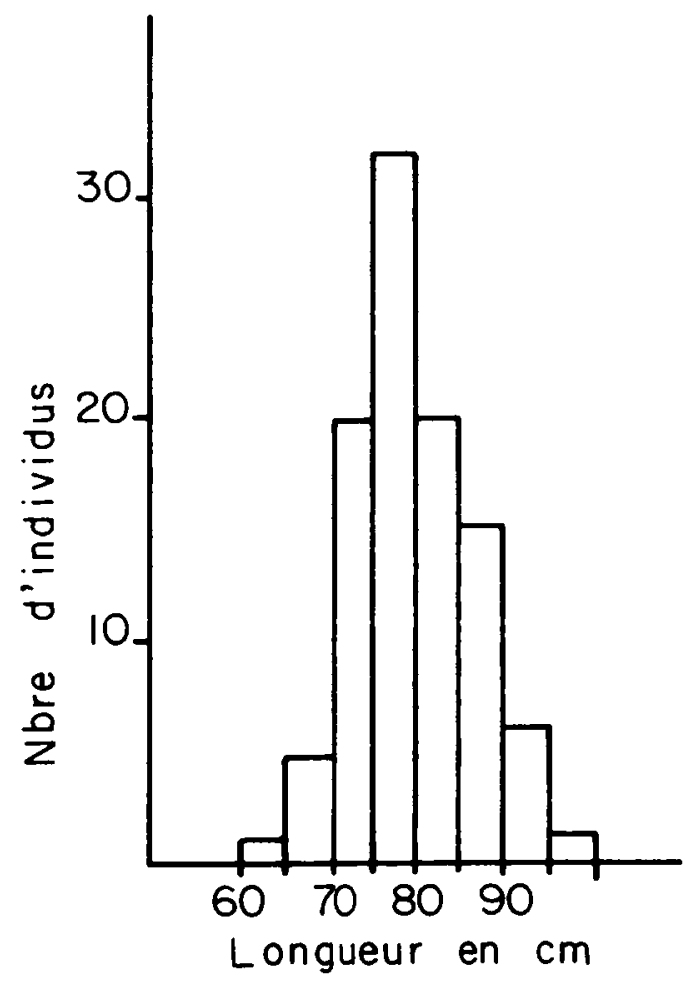

figure 1 : Distribution de la longueur chez la truite fario à l'âge de 6 mois.

figure 1 : Length distribution for 6 months old brown trout.

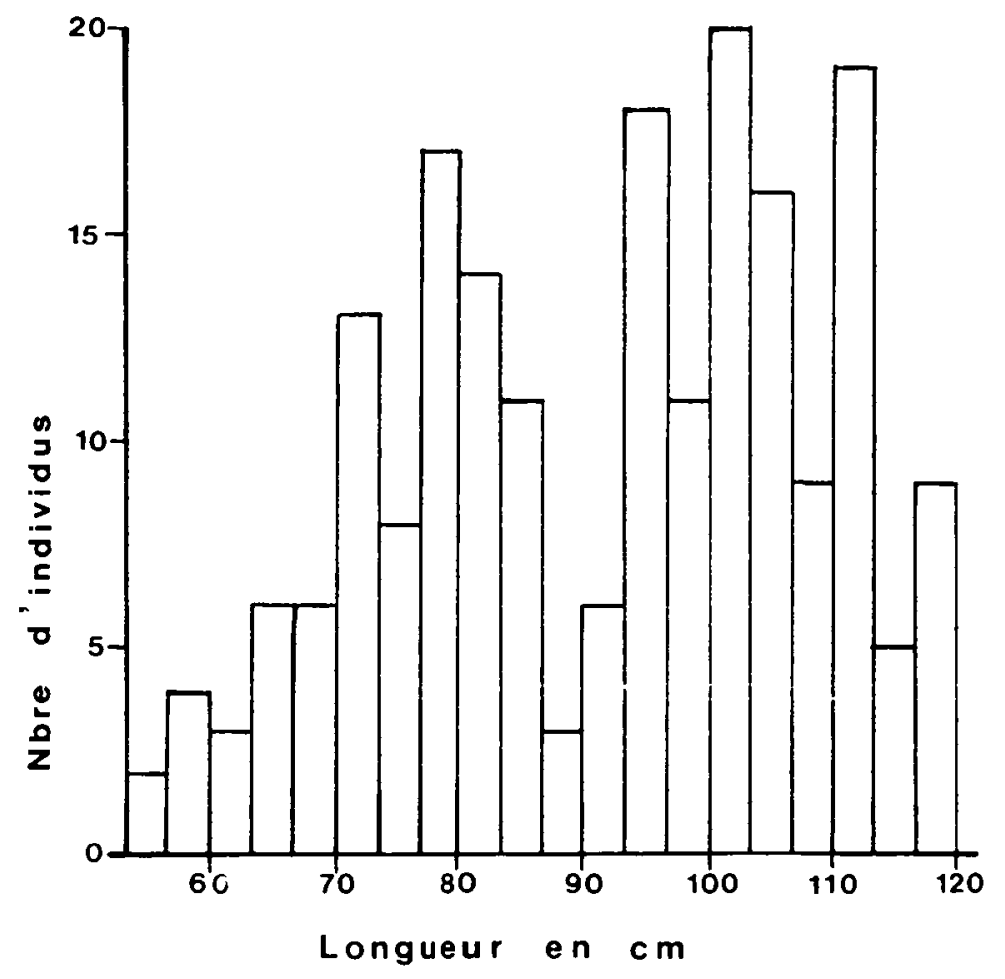

figure 2 : Distribution de la longueur chez le saumon atlantique. Apparition du bimodalisme lié à la smoltification.

figure 2 : Length distribution in atlantic salmon. Bimodalism appears due to smoltification. 


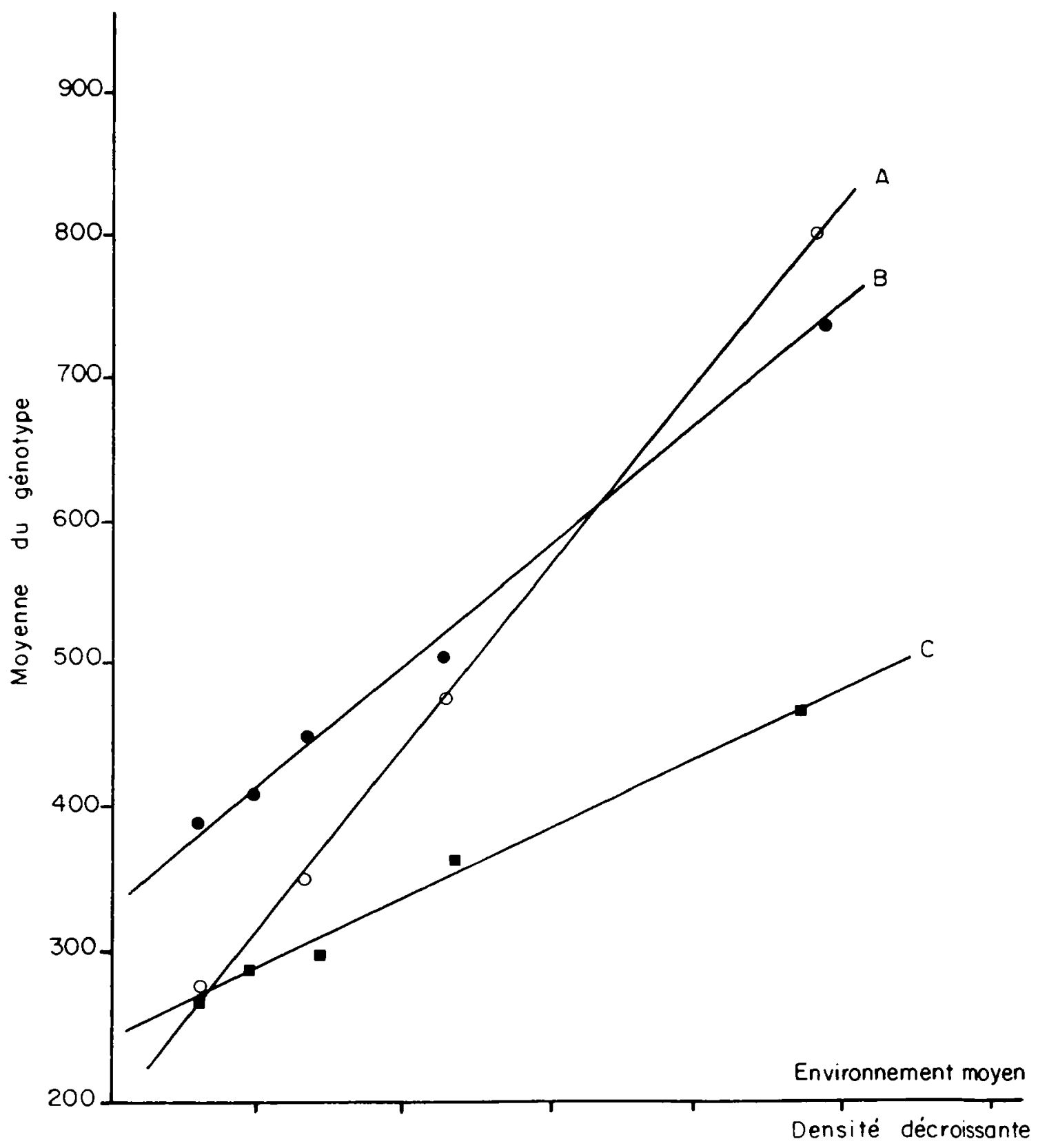

figure 3 : Interaction génotype-environnement chez la carpe (MOAV ef al., 1975).

figure 3 : Genotype-environment interactions in carp (MOAV et al., 1975).

les plantes par multiplication végétative ou chez les mammifères en utilisant des jumeaux vrais. Cela sera sans doute possible dans un avenir proche chez les poissons en utilisant les méthodes d'endomitose (QUILLET, 1987). Ces méthodes permettent dans un premier temps d'obtenir des individus totalement homozygotes. Les descendants d'un mâle et d'une femelle endomitotique seront ainsi génétiquement tous identiques. Une telle étude est actuellement en cours au laboratoire;

- supprimer VE en plaçant les individus dans un milieu aussi homogène que possible: l'expérience montre cependant qu'un milieu considéré comme homogène (par exemple un bassin de pisciculture où tous les animaux sont mélangés) présente encore des hétérogénéités notables. Chez les poissons, une difficulté supplémentaire existe. Il est impossible de les marquer individuellement et de manière précoce (avant 4-5 g). Il est donc impossible de mélanger des lots différents dans un même milieu pour minimiser les effets d'environnement. Une solution consiste 
à avoir des réplicats d'élevage (chaque lot étant élevé dans deux, voire trois bassins différents) ; ce qui permet d'avoir une estimation de la variation due à l'environnement.

Cette séparation stricte $(V G / V E)$ étant impossible, on a recours à des modèles plus complexes qui nécessitent une décomposition plus avancée des effets génétiques et des effets d'environnement.

\section{Effets additifs et non additifs}

Pour définir concrètement les effets additifs, nous reprendrons un exemple dû à SCHMIDT (1919). Pour analyser le déterminisme génétique du nombre de vertèbres, l'auteur part d'un dispositif de 12 lots représentant tous les croisements possibles entre 3 mâles et 4 femelles. Le nombre de vertèbres de ces reproducteurs est connu, mais SCHMIDT souligne que cette valeur (phénotypique) ne permet pas de connaître leur "valeur génétique" $(P=G+E)$. Celle-ci par contre peut être calculée à partir des valeurs moyennes observées dans la descendance. En effet, la moyenne des écarts d'environnement pour une population entière est posée égale à 0 ; la moyenne phénotypique de la population des descendants est donc égale à sa moyenne génotypique. On affecte ainsi à chaque reproducteur une "valeur génétique" définie par la performance moyenne de tous ses descendants (Tableau I). On peut ensuite essayer de prédire la performance d'un croisement donné à partir de la moyenne arithmétique des "valeurs génétiques" des deux reproducteurs utilisés. Si cette prévision est correcte (ce qui peut se tester statistiquement), on parlera d'un déterminisme génétique essentiellement additíf. Par contre, si les performances de certains croisements présentent des écarts notables à la prévision, l'ensemble de ces écarts permettra d'estimer la variation génétique non additive (dite de dominance au sens large).

Complémentairement à cette présentation très globale et purement statistique des effets additifs et non additifs, on peut développer la même réflexion au niveau du mode d'action des gènes impliqués. L'additivité des gènes se trouve respectée lorsque la valeur génotypique de l'hétérozygote est égale à la moitié des valeurs génotypiques des homozygotes. Dans le cas contraire, on parle de dominance (figure 4) (il y a interaction entre les deux gènes d'un même locus) ou de superdominance si la performance de l'hétérozygote est supérieure au meilleur des deux homozygotes. Citons pour exemple de dominance le gène golden chez la truite arc-en-ciel. Ce gène est responsable d'une coloration jaune, et l'hétérozygote $\mathrm{GN}(\mathrm{G}=$ golden, $\mathrm{N}=$ noir) présente le même phénotype jaune que l'homozygote GG.

Pour la plupart des caractères complexes, plusieurs zones en divers points du génome interviennent. Pour cet ensemble, on décompose la valeur génotypique en effets d'additivité et en interactions de dominance avec en plus d'autres types d'interactions inter locus (entre effets additifs, entre effets de dominance ou mixtes) dont la somme constitue les effets d'épistasie. Un exemple biologique serait l'action d'un gène régulateur sur un gène de structure.

Tableau I : Croisement factoriel permettant de calculer les valeurs génétiques des parents.

Table I : Factorial cross from which genetical values of parents are estimated.

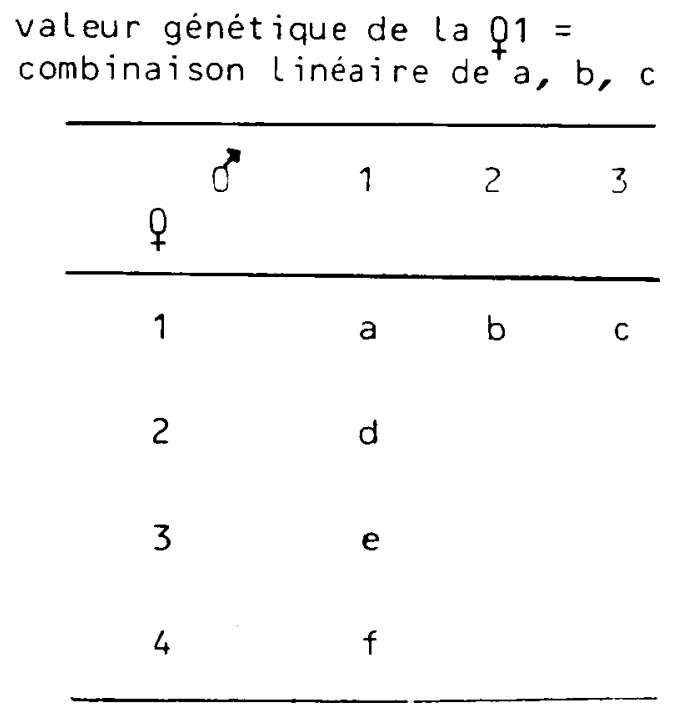

valeur génétique du male $1=$

combinaison Linéaire de $a, d, e, f$. 


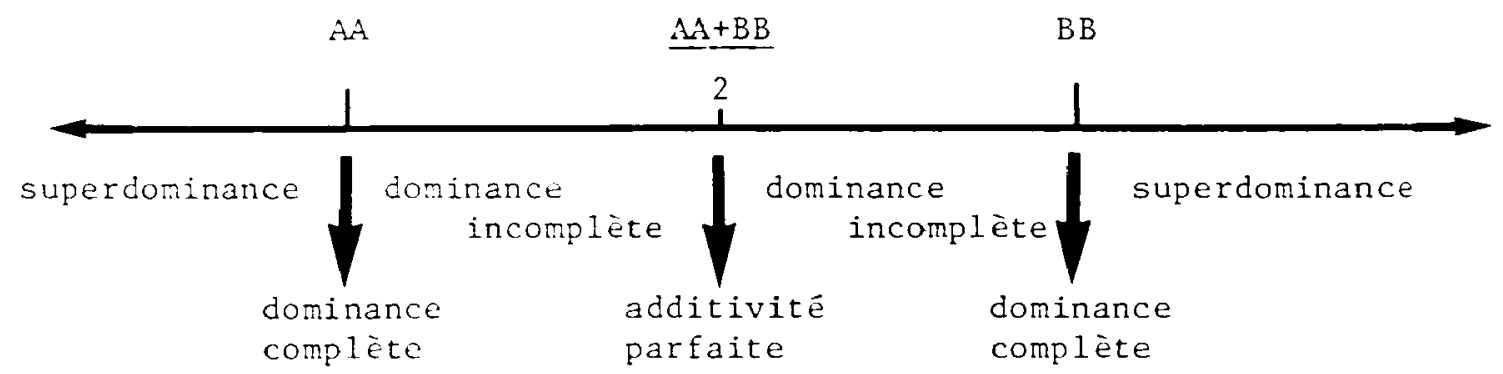

figure 4 : Domaines de définition de l'hétérozygote.

figure 4 : Definition field of the heterozygote.

\section{L'héritabilité}

C'est à partir de la variance génétique additive que l'on estime l'héritabilité :

$h^{2}=\frac{V_{A}}{V_{P}}$

Ce paramètre mesure le degré de ressemblance entre apparentés et a surtout un rôle prévisionnel. C'est en effet principalement à partir de cette valeur que l'expérimentateur établira son schéma d'amélioration génétique.

Nous ne décrirons pas en détail les méthodes de mesure de l'héritabilité, mais notons cependant qu'il est possible:

- soit d'étudier la transmission du caractère en établissant une régression entre les parents et les valeurs moyennes de leurs enfants;

- soit d'analyser la ressemblance entre famille de plein-frères ou de demi-frères. Dans ce cas, les reproducteurs ne servent qu'à la fécondation (citons pour exemple un prélèvement de géniteurs dans une rivière relâchés après récupération des gamètes).

\section{APPLICATION A LA SYSTÉmATIQUE ET A LA BIOGÉOGRAPHIE}

Les caractères quantitatifs utilisés pour définir les espèces, sous-espèces et races locales intègrent aussi bien des effets génétiques que des effets environnementaux. Leur utilisation pour l'étude de la spéciation est donc déconseillée. II faut toutefois noter que les systématiciens ont utilisé des caractères à héritabilité élevée (le nombre de vertèbres, le nombre de caeca pyloriques, la ponctuation rouge et noire), et donc moins sensibles aux effets du milieu. Néanmoins, en toute rigueur, l'héritabilité d'un caractère concerne une population donnée dans un milieu donné souvent le plus homogène possible. Le milieu naturel étant beaucoup plus variable, l'héritabilité peut se trouver fortement diminuée. CHEVASSUS et al., (1979) ont démontré l'influence du facteur thermique sur le nombre de caeca pyloriques. On ne peut donc parler d'héritabilité forte que si elle a été estimée dans différents milieux.

Ainsi, en utilisant ces caractères fortement héritables, les caractères quantitatifs peuvent être de bons guides pour orienter des recherches plus fines. Une sous-espèce de truite fario en Corse, la truite fario macrostigma SPILLMAN (1961) a été décrite sur la base de caractères morphologiques. L'étude du polymorphisme enzymatique KRIEG et GUYOMARD (1983) confirme l'existence d'une importante différenciation gènétique.

\section{APPLICATION A L'AMÉLIORATION GÉNÉTIQUE}

\section{Les critères de sélection}

Dans la gestion d'une souche de repeuplement, on observe deux grandes étapes: a) la phase d'élevage, b) la phase en milieu naturel. Pour chaque étape, il existe des critères de sélection particuliers. Pendant la phase d'élevage, on cherche à améliorer la productivité soit la croissance précoce, la survie, la résistance aux maladies souvent plus présentes en milieu confiné. Pendant la phase en milieu naturel, c'est surtout le taux d'implantation, l'aspect, la rusticité et la survie en milieu sauvage qui sont étudiés.

II est clair que les critères concernant la phase d'élevage sont les plus faciles à manipuler, mais les corrélations génétiques entre les caractères n'étant pas connues, il est difficile de prévoir les effets de sélection en pisciculture sur des animaux destinés au repeuplement. Ainsi G. MAISSE 
(1983), en comparant le taux d'implantation d'une souche hybride (sauvage $x$ domestique) et d'une souche domestique de truite fario, montre que l'importance relative des juvéniles des deux origines apparaît liée aux caractéristiques du milieu naturel. II apparaît donc difficile d'établir une loi générale et des expériences comparatives seront nécessaires.

\section{Les méthodes de sélection}

Deux grandes voies possibles s'offrent à l'expérimentateur

- l'utilisation de la variance génétique additive par l'établissement des schémas de sélection.

- la recherche des effets d'hétérosis par croisement.

a) Amélioration génétique par sélection

Dans la mesure de la réponse à la sélection, trois paramètres interviennent: I'héritabilité du caractère, le taux de sélection, la variabilité phénotypique (sans variabilité, il n'y a pas de sélection possible).

Les deux principales méthodes de sélection utilisables chez les poissons et plus particulièrement chez les salmonidés sont la sélection individuelle ou sélection massale et la sélection combinée.

La sélection individuelle consiste à prendre comme reproducteurs les animaux les plus performants pour le caractère choisi. Cette méthode est particulièrement simple à appliquer et n'implique dans le cas des poissons que peu de contraintes matérielles, notamment tous les reproducteurs comme tous les descendants peuvent être mélangés dans un bassin. De plus, l'importante fertilité des poissons permet des taux de sélection très élevés (de l'ordre de $1 \%$ soit pour 100 géniteurs choisis un démarrage de 100.000 alevins) renforçant de ce fait l'efficacité de la sélection.

La sélection combinée familiale et intrafamiliale consiste à choisir les meilleurs individus parmi les meilleures familles.

La théorie montre que la sélection familiale est préférable à une sélection individuelle quand 1) I'héritabilité du caractère est faible, 2) les familles sont de grande taille, 3) la variation due au milieu commun à tous les membres d'une famille est faible.

Chez les poissons et plus particulièrement les salmonidés, l'héritabilité de la croissance est faible (Tabl. II) et la taille de la famille peut être importante compte-tenu de l'importante fertilité.

\section{Tableau II : Héritabilité de la croissance chez les salmonidés. \\ Table II : Growth heritability in salmonids.}

\begin{tabular}{|c|c|c|c|c|c|}
\hline \multirow[t]{2}{*}{ Espèces } & \multicolumn{3}{|c|}{$h^{2}$} & \multicolumn{2}{|l|}{ RÉfércuces } \\
\hline & 0,16 & $-0,37 \star$ & $1,09-0,29 *$ & Aulstad et $a l$. & $(i 972)$ \\
\hline \multirow[t]{2}{*}{ Salino } & 0,08 & $-0,18$ & $0,04-0,18$ & Chevassus & $(1976)$ \\
\hline & & - & $0,07-0,22$ & Ayles & $(1975)$ \\
\hline \multirow[t]{3}{*}{ gairdneri } & 0 & $-0,5$ & $-0,50$ & Moller et al. & $(1979)$ \\
\hline & 0 & $20 \star$ & $0,06 *$ & Refstie & $(1980)$ \\
\hline & & - & $0,6-0,70$ & Lindroth & $(1972)$ \\
\hline Salmo & 0 , & $12 \star$ & $0,08 \star$ & Refstie et steine & $(1978)$ \\
\hline salar & 0 & $-0,35$ & - & Holm et Naevdal & $(1978)$ \\
\hline
\end{tabular}


La sélection combinée prend en compte la moyenne familiale et la valeur individuelle en les pondérant. Toute chose étant égale par ailleurs, notamment le taux de sélection, la sélection combinée est plus performante. Des gains de poids de 13 à $15 \%$ par génération ont été obtenus chez la truite arc-en-ciel par les norvégiens. Néanmoins, cette méthode impose des contraintes matérielles très importantes. Ainsi, pour obtenir un taux de sélection de $5 \%$ (déjà moins important que celui cité pour la sélection individuelle) et retenir un minimum de 10 familles, il faut en élever 200. Compte-tenu de l'impossibilité d'un marquage individuel précoce, des réplicats d'élevage sont nécessaires et 400 bassins sont ainsi mobilisés. De telles contraintes financières peuvent être difficile à supporter pour un producteur. Actuellement, une procédure d'optimisation de la sélection individuelle est en cours d'étude.

\section{b) Amélioration génétique par croisements}

Les exemples d'hétérosis pour la croissance et la survie sont fréquents chez la carpe (Tabl. III) BAKOS (1987) et constituent la base de schémas d'amélioration génétique développés en Europe de l'est et en Israël. Par contre, peu de résultats intéressants ont été obtenus chez les salmonidés (KLUPP, 1979 ; BAILEY et SAUNDERS, 1979 ; BAILEY et al., 1980 ; GJERDE, 1981 ; LINDER et al., 1983; GJERDE et REFSTIE, 1984). Pour interpréter cette opposition, il faut rappeler que le niveau de différenciation génétique entre les populations croisées est un facteur important pour obtenir des effets d'hétérosis (sans aller toutefois jusqu'à une incompatibilité génétique). Or, il faut remarquer que les différentes populations de carpe ont été maintenues isolées (notamment par la pratique de la reproduction naturelle) et leur grande fertilité a permis une production à partir de peu de géniteurs ; elles ont donc pu diverger gènétiquement de manière importante. Par contre, les populations d'élevages salmonicoles de création plus récente ont subi un important brassage génétique (échange d'œufs, de reproducteurs) et se révèlent peu différenciées entre elles tout en présentant une importante variabilité intrapopulation GUYOMARD (1981), KRIEG et GUYOMARD (1985), explicable par le mélange des populations. Lorsque ce brassage génétique n'a pas été effectué, il est possible d'observer de l'hétérosis. Citons pour exemple le croisement entre deux populations de truites arc-en-ciel isolées depuis plusieurs générations, l'une en provenance de Norvège, l'autre en provenance des USA. Un hétérosis est observé pour la croissance (fig. 5) (données non publiées). Ceci restant une exception, il apparaît nécessaire de créer des lignées plus homogènes et plus différenciées pour détecter d'éventuels effets d'hétérosis. L'obtention de lignées consanguines (différenciées) par les méthodes classiques (autofécondation, croisement frères-sœurs) nécessite plusieurs générations avant tout protocole d'amélioration génétique, handicap rédhibitoire. Aujourd'hui, l'intérêt pour l'amélioration génétique par croisement est relancé car il est possible au niveau expérimental d'obtenir des individus totalement homozygotes en une seule génération. L'étude des recroisements entre de tels individus et la formation des lignées fixées est actuellement en cours au laboratoire.

Tableau III : Hétérosis chez la carpe (données polonaises).

Table III : Heterosis in carp (Polish data).

\begin{tabular}{ccccc}
\hline $\begin{array}{c}\text { Lignes } \\
\text { (souches) }\end{array}$ & $\begin{array}{c}\text { P en } \\
\text { an }(g)\end{array}$ & $\begin{array}{c}\% \\
\text { survie }\end{array}$ & $\begin{array}{c}\text { Production } \\
\mathrm{Kg} / \text { Ha }\end{array}$ & $\begin{array}{c}\text { Coefficient } \\
\text { conversion }\end{array}$ \\
\hline Z & 98 & 57 & 559 & 3,20 \\
G & 140 & 83 & 1169 & 2,85 \\
W & 128 & 90 & 1152 & 2,55 \\
W X Z & 174 & 99 & 1723 & 2,02 \\
W & 162 & 96 & 1555 & 2,13 \\
\hline
\end{tabular}




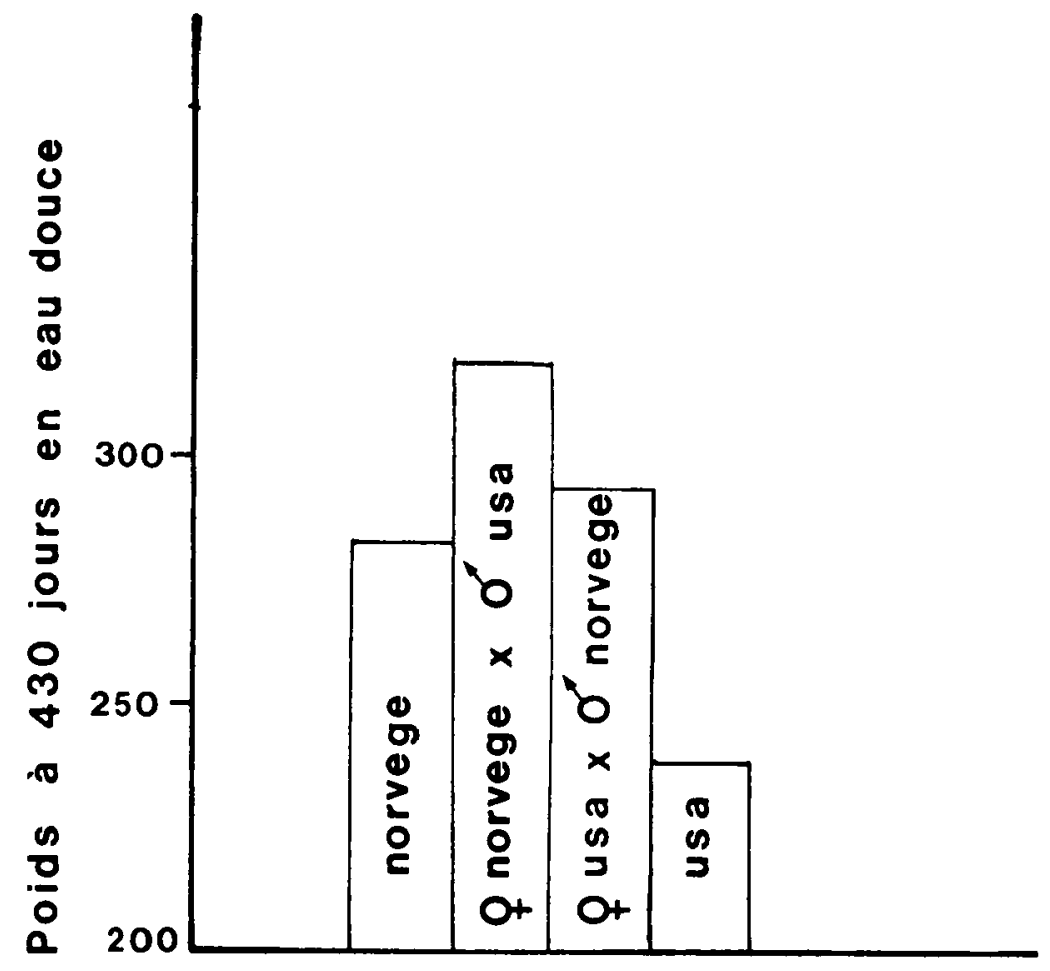

figure 5 : Hétérosis pour la croissance entre deux populations de truites arc-en-ciel en provenance de Norvège et des USA.

figure 5 : Heterosis for growth between two rainbow trout populations from Norway and USA.

\section{CONCLUSION}

S'il ne faut pas idéaliser les populations naturelles et penser que toute amélioration est inutile, il ne faut pas non plus les négliger et les faire disparaître. Aussi, des initiatives basées sur des critères précis, un schéma de sélection efficace et surtout des méthodes de contrôle dans la nature peuvent se révéler intéressantes.

\section{BIBLIOGRAPHIE}

AULSTAD D., GJEDREM T., SKJERVOLD H., 1972. Genetic and environmental sources of variation in length and weight of rainbow trout (Salmo gairdneri). J. Fish. Res. B. Canada, 29, 237-241.

AYLES G.B., 1975. Influence of the genotype and the environment on growth and survival of rainbow trout (Salmo gairdneri) in central canadian aquaculture lakes. Aquaculture, 6. 181-188.

BAILEY J.K. and R.L. SAUNDERS, 1979. Preliminary report on releases and returns of two yearclasses of Atlantic salmon smolts from various pure strains and strain crosses. North American Salmon Research Center Report $n^{\circ} 5,7 \mathrm{p}$.

BAILEY J.K., BUZETA M.I. and R.L. SAUNDERS, 1980. Returns of three year-classes of sea ranched Atlantic salmon of various river strains and their hybrids. North American Salmon Research Center Report $n^{\circ}$ 3, $10 \mathrm{p}$.

BAKOS J., 1987. Selective breeding and intraspecific hybridization of warm water fishes. In: Proc. World Symp. on Selection, Hibridization and Genetic Engineering in Aquaculture, Bordeaux. 27-30 May 1986. Vol. 1: 303-311.

CHEVASSUS B., 1976. Variabilité et héritabilité des performances de croissance chez la truite arc-en-ciel (Salmo gairdneri R.). Ann. Génét. Sél. Anim., 8, 273-283.

CHEVASSUS B., BLANC J.M., BERGOT P., 1979. Déterminisme génétique du nombre de caeca pyloriques chez la truite fario (Salmo trutta L.) et la truite arc-en-ciel (Salmo gairdneri R.). II - Effet du génotype, du milieu d'élevage et de l'alimentation sur la réalisation du caractère chez la truite arc-en-ciel. Ann. Génét. Sél. Anim., 11, 79-92. 
CHOUBERT G. and BLANC J.M., 1985. Flesh colour of diploid and triploid rainbow trout (Salmo gairdneri R.) fed canthaxanthin. Aquaculture, 47, 299-304.

FALCONER D.S., 1960. Introduction to quantitative genetics. Oliver and Boyd, Edimburg, 365 pp.

FISCHER R.A., 1918. The correlation between relatives on the supposition of Mendelian inheritances. Trans. Zoy Soc. Edinb., 52, 399-433.

GJERDE B., 1981. Growth rate and mortality of crosses between strains of Atlantic salmon. 32 nd Annual Meeting of the European Association for animal production, Zagreb, Yougoslavie.

GUYOMARD R., 1981. Electrophoretic variation in four French populations of domesticated rainbow trout (Salmo gairdneri R.) Can. J. Génét. Cytol., 23, 33-47.

HOLM M. and NAEVDAL G., 1978. Quantitative genetic variation in fish. Its significance for salmonid culture. In Marine organisms, B. Battaglia and J. Beardmore ed., Plenum Publishing Corporation. New York, 679-698.

KIRPICHNIKOV V.S., 1981. Genetic bases of fish selection. Springer-Verlag, Berlin Heidelberg, New York.

KLUPP R., Genetic variance for growth in rainbow trout ( $\mathrm{Sa}$ /mo gairdneri L.). Aquaculture, 18, 123-134.

KRIEG F. and GUYOMARD R., 1983. Mise en évidence électrophorétique d'une forte différenciation génétique entre populations de truite fario de Corse. C.R. Acad. Sci., Paris, 296, 1.089-1.094.

KRIEG F. and GUYOMARD R., 1985. Population genetics of French brown trout (Salmo trutta L.) : large geographical differentiation of wild populations and high similarity of domesticated stocks. Génét. Sél. Evol., 17 (2), 225-242.

LINDER D., SUMARI O., NYLHOMK. and SIRKKOMAAS S., 1983. Genetic and phenotypic variation in production traits in rainbow trout strains and strain crosses in Finland. Aquaculture, 33 , 129-134.

LINDROTH A., 1972. Heritability estimates of growth in fish. Aquilo. Sci. Zool., 13, 77-80.

MAISSE G., PORCHER J.P., NIHOUARN A., CHEVASSUS B., 1983. Comparaison des performances en pisciculture d'un hybride intraspécifique ( 0 sauvage $x 0$ domestique) et de la souche domestique chez la truite commune(Salmo trutta). Essais préliminaires d'implantation en ruisseau. Bull. Fr. Pisc., 291, 167-181.

MOAV R., HULATA G. and WOHLFARTH G., 1975. Genetic differences between the chinese and european races of the common carp. I. Analysis of genotype-environment interactions for growth rate. Heredity, 34, 323-340.

MOLLER D., NAEVDAL G., HOLM M., LEROY R., 1979. Variation in growth rate and age of sexual maturity in rainbow trout. In : Advances in Aquaculture, Fernham, 622-626.

QUILLET E., 1987. Amélioration génétique par recroisement de lignées consanguines chez la truite arc-en-ciel. Rapport d'activité GCS-BBA, $13 \mathrm{p}$.

REFSTIE T., 1980. Genetic and environmental sources of variation in body weight and length of rainbow trout fingerlings. Aquaculture, 19, 351-357.

REFSTIE T. and STEINE T. A., 1978. Selection experiments with salmon. III. Genetic and environmental sources of variation in length and weight of atlantic salmon in the freshwater phase. Aquaculture, 14, 221-234.

SCHMIDT J., 1919. La valeur de l'individu à titre de générateur appréciée suivant la méthode du croisement diallèle. C.R. Trav. Lab., Cazlsberg, 14, 1-34.

SPILLMAN C., 1961. Faune de France, poissons d'eau douce. Lechevallier, Paris, $291 \mathrm{p}$. 